\title{
The Atopic March. A Literature Review
}

\author{
Juan F. Salazar-Espinosa.
}

\begin{abstract}
The atopic march is defined as the progression of atopic diseases, generally during childhood, such as atopic dermatitis, asthma, allergic rhinitis and food allergies. The main risk factors for developing these atopic diseases include genetics, aeroallergens, food allergens, late food introduction to the infant, and living in developing countries. The immunologic contributors to this problem include the Tha response, epigenetics, and lack of certain factors like thymic stromal lymphopoietin (TSLP) and filaggrin. As a whole, the therapeutic approach has been changing during recent years because of the discovery of new factors involved in this problem. This article explains the definition of atopic march, the immunological pathway, clinical features, epidemiology and therapeutic approaches to create a context for the broader understanding of this important condition.
\end{abstract}

Keywords: Atopic dermatitis; asthma; allergy; atopy; Th2 response; atopic march (Source: MeSH, NLM)

\section{Introduction}

Atopic march is an epidemiological phenomenon involving atopic diseases and describes the process by which some atopic diseases interrelate through a patient's life. These atopic diseases include asthma, atopic dermatitis (AD), allergic rhinitis, and food allergies. The common progression of these diseases is from atopic dermatitis to asthma and then allergic rhinitis; this is not, however, the only way that this condition progresses. The atopic march has some associated risk factors that have been described in epidemiological studies from different countries. There exist, however, some differences in the disease epidemiology depending on the socioeconomic status of the country studied.'

About the author: Juan F. Salazar-Espinosa is $\mathrm{Cu}$ rrently a 3 rd year medical student of University of Caldas, Manizales, Colombia of a six year program. $\mathrm{He}$ is also the treasurer of the Medical Student's Scientific Association of Universidad de Caldas (ACEMCAL). and thymic stromal lymphopoietin (TSLP) overexpression which
The pathogenesis of the atopic processes share a common systemic Th2 response and epigenetic factors. Related to Th2 response are other immunological factors like filaggrin deficiency have been demonstrated in mouse models and serve as therapeutic targets. ${ }^{2}$ These exist in addition to the ratio between histone acetyl transferase (HAT) and histone deactetylase (HDAC) as the most important epigenetic factors involved in allergies. ${ }^{3}$ The therapeutic approach has multiple pharmacological options, including subcutaneous and sublingual immunotherapy, prebiotics, probiotics and, most recently, anti-IgE monoclonal antibodies. The multitude of therapeutic approaches seen in atopic march may be explained by the observation that there are different preferences for various treatments among different medical specialties. ${ }^{4}$

\section{Search Strategy and Selection Criteria}

A literature search was performed using MEDLINE MeSH ter ms "eczema", "asthma", "food allergy" and "allergic rhinitis" where each term was combined with "atopic march" using the PubMed search builder. A total of 177 articles were found. Following filtering by author criteria (publication within last 20 years, English language, and the existence of a relation between at least two atopic diseases), 32 articles remained and an additional 22 articles were retrieved from references. This review follows the Preferred Reporting Items for Systematic Reviews and Meta-Analyses (PRISMA) Statement. ${ }^{5}$

\section{Immunological Pathway}

The first step in atopic march pathogenesis is allergen exposure or the first sensitization episode in a patient's life, leading to the activation of the epithelial cell triggers and the release of TSLP and tumor necrosis factor alpha (TNF- $\alpha$ ). These are chemotactic factors for dendritic cells, which induce the expression of adhesion molecules at the endothelium. ${ }^{6}$ Dendritic cells internalize the allergen through their high affinity IgE receptors (FcERI) and migrate and mediate the naïve T cell activation in Th1 cells, which releases interleukin-2 (IL-2) and interferon-gamma (IFN- $\gamma$ ), and Th2 cells, which releases IL-4, in the lymph nodes. Following this is a process called scratch-rubbing cycle where a skin barrier dysfunction (filaggrin mutations) leads to a decrease in Th1 response and an increase in Th2 response with the release of IL-5 and IL-13. This triggers the increase in eosinophilic infiltration with an immunoglobulin E (IgE) hyperproduction. The Th2 response enhances the allergen response in the nasal and bronchial mucosa, leading to eosinophil infiltration and IgE hypersecretion in addition to the mast cell proliferation, epithelial cell activation, mucus hypersecretion, and smooth muscle proliferation observed in asthma. ${ }^{78}$ 
Filaggrin is a protein produced in the stratum corneum of the epidermis that plays an essential role in the formation of the protective skin barrier by the prevention of water loss and entry of microbes into the inner layers of the skin. The main mechanism that involves filaggrin in the allergic disease pathogenesis is the enhancement of the Th2 systemic response, which leads to the susceptibility of skin, bronchial, nasal, and gastrointestinal epithelia to the development of atopic diseases, especially atopic dermatitis and asthma. ${ }^{9}$ Importantly, it has been discovered that the maintenance and repair of the epidermal barrier in infants with $A D$ may prevent the subsequent development of asthma. ${ }^{10}$

The TSLP is an IL-7-like interleukin which is produced in the skin, gut, lungs and thymus and contributes to the activation of dendritic cells without the allergen molecule, the predomination of Th2 differentiation, and the upregulation of this response by the mast cells in the nasal epithelium, leading to the development of allergic rhinitis." Figure 1 shows the process involved in the generation of atopic march and some of the factors that may contribute to the development of atopic diseases.

Takai et al., suggested that environment, infection, and/or self-derived toll-like receptor ligands contribute to the initiation and/or amplification of Th2-type skin inflammation, including atopic dermatitis, through the induction of TSLP expression in keratinocytes. This finding is helpful for understanding the role of the gene-environment interaction relevant to allergic diseases. ${ }^{12}$

One of the main factors in the epigenetics of the atopic march is GATA-3, which is activated by T-cell receptor (TCR) and IL-4 receptor activation and maintains its own expression through positive feedback. GATA-3 induces permissive histone and chromatin changes at the Th2 Locus Control Region (LCR) which enhances the Th2 response and suppresses the Th1 response against the allergens. Another important factor is FFOXP3, which regulates the $T$ regulator cell action by promotiing the binding of demethylation factors and the consequent lack of toleration to self and exogenous antigens. ${ }^{3}$

\section{Mouse Models in Atopic March}

Zhang et al., carried out investigations in which they injected mice with intra-peritoneal ovalbumin and measured the TSLP produced by keratinocytes. TSLP is the mediator of the skin inflammation triggered by an allergen and consequent sensitization. Additionally, another mouse model demonstrates a relation between high skin keratinocyte-derived TSLP, high TSLP serum levels and the upregulated epicutaneous sensitization, and aggravated allergic asthma generated upon allergen challenge in airways. ${ }^{13,14}$ However, Demrhi et al., demonstrated that during the first 3 years of life, the plasma circulating TSLP of children with eczema, allergen sensitization, or wheezing was not statistically associated with the manifestation of these symptoms. ${ }^{2}$ The TSLP is implicated in mouse models for the development of atopic diseases. This supports the utilization of TSLP inhibition as a proposed therapeutic option to prevent or limit allergen sensitization and thus halt the progress of the atopic march. ${ }^{15}$

\section{Clinical Features \\ Atopic Dermatitis}

There are three established phases in the progression of $A D$ : an infantile phase (from birth to 2 years of age), a childhood phase ( 2 years to puberty) and the adult phase (from puberty to the adulthood). The first manifestations correspond to erythematous papules and vesicles that typically begin on the face and are intensely pruritic. There is also edema of affected areas, leading to oozing and crusting. The childhood phase of $A D$ additionally presents with lichenified papules and plaques, representing a more chronic disease. Lymphadenopathy might be a prominent feature in affected children. Although the disease sometimes tends to self-resolve, there is an adult phase of $A D$ which predominantly involves the flexural folds, the face and neck, the upper arms and back, and the dorsa of the hands, feet, fingers, and toes. Weeping, crusting, and exudation might occur, usually as a result of superimposed Staphylococcal infection. ${ }^{10}$

\section{Asthma, Allergic Rhinitis and Food Allergy}

A wide array of risk and protective factors including hygiene, infections, outdoor and indoor air pollution, allergen exposure, breast-feeding practices, genetic factors, nutrition, and obesity play a multifaceted role in shaping the observed worldwide trends of respiratory allergies. ${ }^{16}$ Some of these patients never present with any clinical symptoms throughout their lives, although airway hyperresponsiveness, shortness of

Figure 1. A proposed pathway to explain the atopic march from childhood eczema to the development of asthma and allergic rhinitis. ${ }^{4}$

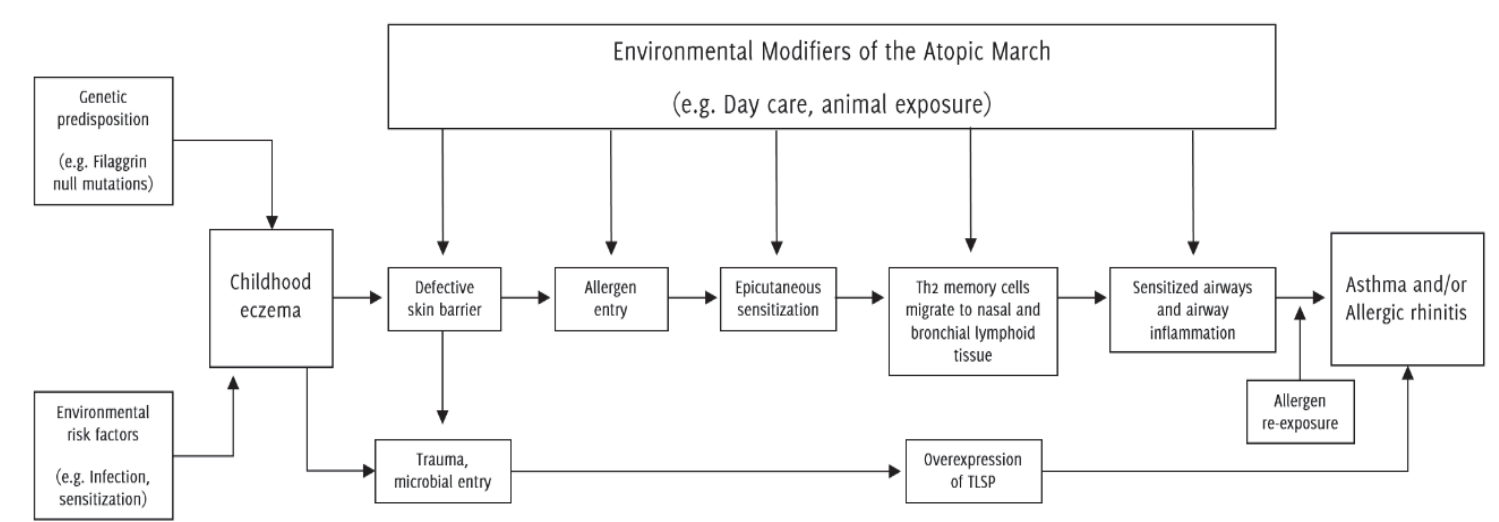

Source: Dharmage SC, Lowe AJ, Matheson MC, Burgess JA, Allen KJ, Abramson MJ. Atopic dermatitis and the atopic march revisited. Allergy. 2014 Jan;69(1):17-27. Reprinted with permission from John Wiley and Sons. 
breath, wheezing which causes chronic airflow limitation, and decreased lung function are common. Examinations of these patients reveal low forced expiratory volume in 1 second $\left(\mathrm{FEV}_{1}\right)$ together with an increased eosinophil and Th2 infiltration in bronchial tissue. An Indian study shows that fractional exhaled nitric oxide (FeNO) is a marker of lower airway inflammation; therefore, significantly higher FeNO levels in atopic allergic rhinitis patients may predict the onset of asthma. ${ }^{17}$ For allergic rhinitis, there are four main symptoms: watery rhinorrhea, nasal obstruction, nasal itching, and sneezing..$^{18}$ Like asthma, allergic rhinitis could be classified as mild or moderate-severe based on severity. ${ }^{19-21}$

Food allergy is known to be one part of this atopic process and its incidence tends to be higher at 2 years of life. ${ }^{22} \mathrm{~A}$ clinically relevant event is cow milk allergy, which is proposed as a risk factor for the development of asthma, rhinoconjuctivitis and eczema. ${ }^{23}$ Food allergy is clinically well characterized by urticaria, angioedema, vomiting or anaphylaxis. These clinical manifestations are present within two hours following the food ingestion. There are some foods that mediate this disease like peanuts, cow milk, and eggs. Besides these symptoms other clinical manifestations include a scratchy throat, hives anywhere on the body, swelling of the eyelids, face, or tongue, nausea, cramps, vomiting, diarrhea, nasal congestion, and shortness of breath or wheezing due to the exposure to a hapten of the food. ${ }^{24}$ A controversial dilemma, which began in the 1970 s and remains unresolved, includes whether the introduction of food antigens into the diet either early or later in life has any effect on the development of food allergy, atopy or allergic asthma. This is explained by the antigens present in the food (cow milk) which are captured by the Peyer patches and generate a Th1 response in healthy children. ${ }^{25,26}$ Some studies concluded that the early food introduction and six months breastfeeding after birth are poor prognostic factors and predispose the infants to the development of food allergy. ${ }^{27}$ However, a population-based prospective cohort study using 3781 children performed in 2013 established that long term breastfeeding and the early introduction (with specific timing) of wheat, rye, oats, barley cereals, fish, and eggs is preferred in that it protects against the development of asthma and allergic rhinitis from a few months following birth to the age of five. This finding suggests a potential conflicting effect of breastfeeding on different asthma phenotypes..$^{28}$

\section{Epidemiology}

There are three established progressive pathways in this process: (1) atopic dermatitis to asthma and allergic rhinitis, (2) asthma to atopic dermatitis, and (3) asthma to rhinitis without the presence of atopic dermatitis. Food allergy is an associated risk factor in the development of these three conditions, and for this reason it can be found in any phase of the atopic march. However, a reverse atopic march has been proposed, with patients developing asthma first with the later appearance of atopic dermatitis. This is demonstrated in a prospective study that followed nearly 700 children aged six to nine years with asthma alone for nine years. Twenty percent of the children developed atopic dermatitis at the end of nine years. ${ }^{29}$

Atopic dermatitis has a prevalence that ranges from $7 \%$ to $30 \%$ in children and from $2 \%$ to $10 \%$ in adults. ${ }^{30} \mathrm{AD}$ and food aller- gies have the highest incidence in the first two years of life. Sensitization to inhalant allergens is rare at that time of life. In later childhood, the prevalence of $A D$ and food allergies decreases while the prevalence of asthma, allergic rhinitis, and sensitization to inhalant allergens rises. ${ }^{31}$ Shen et al., followed a birth cohort for seven years and classified the eczema into preschool and late-onset and examined the correlation between the development of asthma and allergic rhinitis. Results showed that the 36 month old group (late-onset group) had the highest risk for developing asthma or allergic rhinitis five years later. This indicates that it is necessary to evaluate the associated risk factors at the first presentation of atopic disease in order to predict its progression in subsequent years. ${ }^{32}$

A longitudinal study of a prospective birth cohort of 620 children described a higher prevalence of atopic march in boys than girls by a mechanism that remains unknown. ${ }^{33}$ Higher body mass index (BMI) is a risk factor for atopy, wheezing, and cough in girls only. ${ }^{34}$ However, higher BMI is not a risk factor for asthma or airway hyperresponsiveness in either boys or girls. As the atopic march is a disease of interrelation, the etiology is not the same for all components. In fact, it is described that parents with asthma give birth to children with four times the odds of developing food allergy. ${ }^{35}$ The food allergy is associated with six times the odds of developing asthma compared to patients without any food allergy. ${ }^{36,37}$

It is believed that developed countries have reached a plateau in the incidence of asthma while in developing countries the incidence of these diseases is still increasing. ${ }^{16}$ Van der Hulst et al., conducted a systematic review which assessed the risk of developing asthma in children with atopic eczema during the first four years of life and found that the prevalence of asthma at the age of six years in eczema cohort studies was $35.8 \%$ for inpatients and $29.5 \%$ for a combined group of inpatients and outpatients. ${ }^{38}$ A Norway study, with 4780 children divided into two groups depending on whether they were followed from two years old to six years old $(n=2192)$ or not followed $(n=2588)$. Among children with follow-up data at six years, the prevalence of eczema, asthma, and wheezing at two years was $17.8 \%$, $6.5 \%$ and $25.3 \%$, respectively. Among children with follow-up data at six years, the odds ratio between eczema at two years and current asthma at six years was 1.95 . When the age of onset for eczema was included in the model, an onset before four months of age was significantly associated with asthma at six years $(O R=4.51) .39$

Meanwhile, in middle-income countries, a study conducted during the 2009-2010 period in Colombia collected information from 5,978 patients and analyzed three principal atopic diseases: allergic rhinitis, atopic eczema and asthma. It was discovered that $12 \%$ of the patients had asthma, $32 \%$ had allergic rhinitis and $14 \%$ had atopic eczema and demonstrated an increased presentation of symptoms characteristic of these conditions. ${ }^{40}$ In Taiwan, Hwang CY et al., collected longitudinal cohort information through the medical institution databases that followed patients for eight years. Overall, 66,446 patients were diagnosed with atopic dermatitis and $49.8 \%$ of them had concomitant allergic rhinitis and/or asthma. The overall 8-year prevalence of atopic dermatitis, allergic rhinitis, and asthma was $6.7 \%, 26.3 \%$ and $11.9 \%$, respectively. ${ }^{41}$ In addition, Waked 
et al., administered standardized written questionnaires to 5 to 12-year-old students at 22 schools. In total, 3,909 individuals were analyzed. The prevalence of diagnosed asthma ( $4.8 \%)$ was low, while the prevalence of allergic rhinitis was $21.2 \%$ and that of eczema was $11.8 \%$. Marked variations and differences were found across the governorates in Lebanon, with the lowest prevalence of diagnosed asthma found in Bekaa (1.8\%) and the highest prevalence in Beirut (11.6\%). ${ }^{42}$ The differences in the prevalence of atopic diseases between developing and developed countries likely reflect the differences in the extent of urbanization, industrialization, life style, latitude, and disease severity. Other possible factors, such as socioeconomic status or ethnic group, may also contribute to this variation.

\section{Therapeutic Approaches}

Gordon BR et al., suggested that it is possible to interrupt the atopic march to prevent allergy, especially asthma, with three possible interventions: (1) supplements of dietary probiotics, (2) exclusive breast feeding during the first few months of life, which provides protection from the development of allergies through the high transforming growth factor beta (TCF-B) levels in breast milk, and (3) treatment with inhalant allergen immunotherapy by either subcutaneous or sublingual immunotherapy, both of which decrease the risk of progression to asthma and allergic rhinitis. From current data, if these strategies were widely employed, there would likely be a substantial reduction in future asthma cases. ${ }^{43}$ This is supported by the 2013 PRACTALL trial, which has established a subcutaneous immunotherapy and a sublingual immunotherapy for allergic asthma and rhinitis based on scores given for symptom reduction, improvement in quality of life, and the induction of favorable changes in specific immunologic markers. ${ }^{44}$ In a randomized controlled trial, Passalacqua et al., found immunotherapy for allergic rhinitis to be a preventive factor for asthma development due to the impairment of new sensitizations. ${ }^{21}$

Anti-IgE treatment with omalizumab is another therapeutic option used for rhinitis where its mechanism of action is by specifically binding to IgE with the consequent interruption in the allergic response. New information suggests that the combination of immunotherapy with omalizumab is a good strategy to treat allergic rhinitis. ${ }^{45}$ However, when the monoclonal antibody XmAb7195, human leukocyte-engrafted mouse like omalizumab, was administered, it selectively reduced serum human IgE levels and was much more effective than omalizumab in suppressing IgE levels by dual pathways involving IgE neutralization and a CD32b-dependent inhibition of the formation of IgE-secreting plasma cells. This demonstrates new advances in therapeutic approaches by inhibiting one of the main factors in the development of atopic diseases (i.e. IgE)..$^{46}$

Prebiotics, probiotics and breast feeding balance the "Th2 bias", thus improving intestinal processing of antigens ingested in the diet and reducing intestinal inflammation and IgE production by increasing the uptake of antigens by Peyer's patches. This prevents the development of a Th2-exaggerated immune response which would lead to the systemic sensitization and major enhancement and distribution to tissues like bronchial tissue. ${ }^{47}$ Breastfeeding is recommended to be complemented with probiotics because it increases the number of of immunoglobulin secretor cells, which is one of the main factors involved in protection against atopic problems. ${ }^{4}$ It is theorized that probiotics act at the Clostridium/Bifidobacterium ratio by increasing the Bifidobacterium populations. ${ }^{49}$ Moreover, according to Vitaliti et al., the use of probiotics induces the enhancement of regulatory cell responses to produce more anti-inflammatory cytokines which may decrease the likelihood of the development of allergies. ${ }^{50}$ In 62 mother-infant pairs, Lactobacillus rhamnosus GG administered during the four weeks before the infant's birth and during breast feeding (first three months) increased the immuno-protective potential of breast milk, as assessed by the enhancement of anti-inflammatory TGF-B2 in the milk of the mothers receiving probiotics vs. placebo..$^{51}$ Furthermore, the probiotics are considered to be the main factors involved in the prevention of atopic march. ${ }^{52}$ Prebiotics are nondigestible food products (most commonly oligosaccharides) that stimulate the growth or activity of $\mathrm{Bi}$ fidobacteria and other "friendly" bacteria in the colon. However, further research is needed before routine use of prebiotics can be recommended for prevention of allergy in formula fed infants. It is unclear whether the use of prebiotic should be restricted to infants at high risk of allergy or whether they may have an effect in low risk populations or on other allergic diseases including asthma. ${ }^{53}$

\section{Conclusion}

New information constantly expands our understanding of allergen-induced diseases; this information is reflected in epidemiological studies which demonstrate a non-linear development and relationship of the various components of the atopic march. The atopic march should be understood as an immunological imbalance in Th2 responses together with factors like the TSLP, lack of filaggrin, and epigenetics (FOXP3 and (ATA-3). The epidemiologic data tends to be in accordance with data obtained using mouse models, which provide insights to the development of additional epidemiologic studies. In regard to the therapeutic approach, atopic march should always be understood as a multiple-disease problem and not simply as a single atopic disease. The monoclonal antibodies, sublingual therapy, immunotherapy, prebiotics and probiotics are proving to be promising therapies which need further investigation to demonstrate their action against the immunological factors involved in this disease. 


\section{References}

1. Shaker M. New insights into the allergic march. Curr Opin Pediatr. 2014 Aug;26(4):516-20.

2. Demehri S, Yockey LJ, Visness CM, Jaffee KF, Turkoz A, Wood RA, et al. Circulating TSLP associates with decreased wheezing in non-atopic preschool children: Data from the URECA birth cohort. Clin Exp Allergy. 2014 Jun;44(6):851-7. 3. Begin P, Nadeau KC. Epigenetic regulation of asthma and allergic disease. Allergy Asthma Clin Immunol. 2014 May 28;10(1):27.

4. Dharmage SC, Lowe AJ, Matheson MC, Burgess JA, Allen KJ, Abramson MJ. Atopic dermatitis and the atopic march revisited. Allergy. 2014 Jan;69(1):17 27.

5. Liberati A, Altman DG, Tetzlaff J, Mulrow C, Gøtzsche PC, Loannidis JP, et al. The PRISMA statement for reporting systematic reviews and meta-analyses of studies that evaluate health care interventions: explanation and elaboration. PLOS Med. 2009 Jul 21;6(7):e1000100.

6. Akdis CA, Akdis M, Bieber T, Bindslev-Jensen C, Boguniewicz M, Eigenmann $P$, et al. Diagnosis and treatment of atopic dermatitis in children and adults: European Academy of Allergology and Clinical Immunology/American Academy of Allergy, Asthma and Immunology/PRACTALL Consensus Report. J Allergy Clin Immunol. 2006 Jul;118(1):152-69.

7. Spergel JM, Paller AS. Atopic dermatitis and the atopic march. Allergy Clin Immunol. 2003 Dec;112(6 Suppl):S118-27.

8. Spergel JM. Atopic march: link to upper airways. Curr Opin Allergy Clin Immunol. 2005 Feb;5(1):17-21.

9. Marenholz I, Nickel R, Ruschendorf F, Schulz F, Esparza-Gordillo J, Kerscher $T$, et al. Filaggrin loss-of-function mutations predispose to phenotypes involved in the atopic march. J Allergy Clin Immunol. 2006 0ct;118(4):866-71.

10. Zheng T, Yu J, Oh MH, Zhu Z. The atopic march: progression from atopic dermatitis to allergic rhinitis and asthma. Allergy Asthma Immunol Res. 2011 Apr;3(2):67-73.

11. He R, Geha RS. Thymic stromal lymphopoietin. Ann N Y Acad Sci. 2010 Jan;1183:13-24.

12. Takai T, Chen X, Xie Y, Vu AT, Le TA, Kinoshita H, et al. TSLP Expression Induced via Toll-Like Receptor Pathways in Human Keratinocytes. Methods Enzymol. 2014;535:371-87.

13. Zhang Z, Hener P, Frossard N, Kato S, Metzger D, Li M, et al. Thymic stromal lymphopoietin overproduced by keratinocytes in mouse skin aggravates experimental asthma. Proc Natl Acad Sci U S A. 2009 Feb 3;106(5):1536-41.

14. Auriemma M, Vianale $G$, Amerio P, Reale M. Cytokines and T cells in atopic dermatitis. Eur Cytokine Netw. 2013 Mar;24(1):37-44.

15. Li M. Skin TSLP and "atopic march": What do we learn from mouse mo dels?. Rev Fr Allergol. 2012 Jun;52(4):324-6.

16. Hatzler L, Hofmaier S, Papadopoulos NG. Allergic airway diseases in childhood - marching from epidemiology to novel concepts of prevention. Pediat Allergy Immunol. 2012 Nov;23(7):616-22.

17. Kumar R, Gupta N, Goel N. Correlation of atopy and FeNO in allergic rhinitis: an Indian study. Indian J Chest Dis Allied Sci. 2013 Apr-Jun;55(2):79-83. 18. Min YG. The pathophysiology, diagnosis and treatment of allergic rhinitis. Allergy Asthma Immunol Res. 2010 Apr;2(2):65-76.

19. Bousquet J, Annesi-Maesano I, Carat F, Leger D, Rugina M, Pribil C, et al. Characteristics of intermittent and persistent allergic rhinitis: DREAMS study group. Clin Exp Allergy. 2005 Jun;35(6):728-32.

20. Liu CY, Zhang Y, Han DM, Zhang L. Evaluation of serum specific IgE fo the diagnosis of allergic rhinitis with multi-allergens. Chin Med J (Engl). 2010 0ct;123(20):2836-41.

21. Passalacqua G, Durham SR. Allergic rhinitis and its impact on asthma up date: allergen immunotherapy. J Allergy Clin Immunol. 2007 Apr;1 19(4):881-91 22. Schroeder A, Kumar R, Pongracic JA, Sullivan CL, Caruso DM, Costello J et al. Food allergy is associated with an increased risk of asthma. Clin Exp Allergy. 2009 Feb;39(2):261-70.

23. Noh G, Lee JH. Revision of immunopathogenesis and laboratory inter- pretation for food allergy in atopic dermatitis. Inflamm Allergy Drug Targets. 2012 Feb;11(1):20-35

24. Luo J, Li Y, Gong R. The mechanism of atopic march may be the 'social' event of cells and molecules (Review). Int J Mol Med. 2010 Dec;26(6):779-85. 25. Isolauri E, Arvola T, Sutas $Y$, Moilanen E, Salminen S. Probiotics in the management of atopic eczema. Clin Exp Allergy. 2000 Nov;30(11):1604-10. 26. MacDonald TT, Di Sabatino A. The immunologic basis for gastrointestinal food allergy. Curr Opin Gastroenterol. 2009 Nov;25(6):521-6.

27. Nwaru BI, Takkinen HM, Niemela O, Kaila M, Erkkola M, Ahonen S, et al. Timing of infant feeding in relation to childhood asthma and allergic diseases. J Allergy Clin Immunol. 2013 Jan;131(1):78-86.

28. Szajewska $\mathrm{H}$. The prevention of food allergy in children. Curr Opin Clin Nutr Metab Care. 2013 May;16(3):346-50.

29. Barberio G, Pajno GB, Vita D, Caminiti L, Canonica CW, Passalacqua G. Does a 'reverse' atopic march exist?. Allergy. 2008 Dec;63(12):1630-2.

30. Spergel JM. From atopic dermatitis to asthma: the atopic march. Ann Allergy Asthma Immunol. 2010 Aug;105(2):99-106; quiz 107-9, 117.

31. Kijima A, Murota H, Takahashi A, Arase N, Yang L, Nishioka M, et al. Prevalence and impact of past history of food allergy in atopic dermatitis. Allergol Int. 2013 Mar;62(1):105-12.

32. Shen CY, Lin MC, Lin HK, Lin CH, Fu LS, Fu YC. The natural course of ecze ma from birth to age 7 years and the association with asthma and allergic rhinitis: a population-based birth cohort study. Allergy Asthma Proc. 2013 Jan-Feb;34(1):78-83.

33. Lowe AJ, Carlin JB, Bennett CM, Hosking CS, Abramson MJ, Hill DJ, et al. Do boys do the atopic march while girls dawdle? J Allergy Clin Immunol. 2008 May;121(5):1190-5.

34. Schachter LM, Peat JK, Salome CM. Asthma and atopy in overweight children. Thorax. 2003 Dec;58(12):1031-5.

35. American College of Allergy, Asthma, ct Immunology. Food allergy: a practice parameter. Ann Allergy Asthma Immunol. 2006 Mar;96(3 Suppl 2):S1-68. 36. Allen KJ, Dharmage SC. The role of food allergy in the atopic march. Clin Exp Allergy. 2010 Oct;40(10):1439-41

37. Spergel JM. Epidemiology of atopic dermatitis and atopic march in children. Immunol Allergy Clin North Am. 2010 Aug;30(3):269-80.

38. Van der Hulst AE, Klip H, Brand PL. Risk of developing asthma in young children with atopic eczema: a systematic review. J Allergy Clin Immunol. 2007 Sep;120(3):565-9.

39. Saunes M, Oien T, Dotterud CK, Romundstad PR, Storro 0, Holmen TL, et al. Early eczema and the risk of childhood asthma: a prospective, population-based study. BMC Pediatr. 2012 Oct 24;12:168.

40. Dennis R, Caraballo L, García E, Rojas M, Rondon M, Pérez A, et al. Pre valence of asthma and other allergic conditions in Colombia 2009-2010: a cross-sectional study. BMC Pulm Med. 2012 Jul 13;12:17

41. Hwang CY, Chen YJ, Lin MW, Chen TJ, Chu SY, Chen CC, et al. Prevalence of atopic dermatitis, allergic rhinitis and asthma in Taiwan: a national study 2000 to 2007. Acta Derm Venereol. 2010 Nov;90(6):589-94.

42. Waked M, Salameh P. Asthma, allergic rhinitis and eczema in 5-12-year old school children across Lebanon. Public Health. 2008 Sep;122(9):965-73.

43. Gordon BR. The allergic march: can we prevent allergies and asthma?. Otolaryngol Clin North Am. 2011 Jun;44(3):765-77, xi.

44. Tan RA, Corren J. The relationship of rhinitis and asthma, sinusitis, food allergy, and eczema. Immunol Allergy Clin North Am. 2011 Aug;31(3):481-91. 45. Patrizi A, Pileri A, Bellini F, Raone B, Neri I, Ricci G. Atopic Dermatitis and the Atopic March: What Is New?. J Allergy (Cairo). 2011;2011:279425.

46. Bochner BS, Rothenberg ME, Boyce JA, Finkelman F. Advances in mechanisms of allergy and clinical immunology in 2012. J Allergy Clin Immunol. 2013 Mar;131(3):661-7.

47. Van Bever HP, Samuel ST, Lee BW. Halting the allergic march. World Allergy Organ J. 2008 Apr;1(4):57-62.

48. Rinne M, Kalliomaki M, Arvilommi H, Salminen S, Isolauri E. Effect of 


\section{Review}

probiotics and breastfeeding on the bifidobacterium and lactobacillus/ enterococcus microbiota and humoral immune responses. J Pediatr. 2005 Aug;147(2):186-91.

49. Thomas DW, Greer FR; American Academy of Pediatrics Committee on Nutrition; American Academy of Pediatrics Section on Gastroenterology, Hepatology, and Nutrition. Probiotics and Prebiotics in Pediatrics. Pediatrics. 2010 Dec;126(6):1217-31.

50. Vitaliti G, Pavone P, Guglielmo F, Spataro G, Falsaperla R. The immuno-

modulatory effect of probiotics beyond atopy: an update. J Asthma. 2014
Apr;51(3):320-32.

51. Rautava S, Kalliomaki M, Isolauri E. Probiotics during pregnancy and breast-feeding might confer immunomodulatory protection against atopic disease in the infant. J Allergy Clin Immunol. 2002 Jan;109(1):119-21.

52. Giudice MM, Rocco A, Capristo C. Probiotics in the atopic march: highlights and new insights. Dig Liver Dis. 2006 Dec;38 Suppl 2:S288-90.

53. Osborn DA, Sinn JK. Prebiotics in infants for prevention of allergy. Cochrane Database Syst Rev. 2013 Mar 28;3:CDo06474.

\section{Acknowledgments}

None.

Conflict of Interest Statement it Funding

The author has no funding, financial relationships or conflicts of interest to disclose.

\section{Author Contributions}

Conception and design the work/idea, Write the manuscript, Critical revision of the manuscript, Approval of the final version: JFSE.

Cite as:

Salazar-Espinosa JF. The Atopic March. A Literature Review. Int J Med Students. 2014 Jul-0ct;2(3):119-24. 